\title{
Common plants as alternative analytical tools to monitor heavy metals in soil
}

\author{
Daniela Malizia, Antonella Giuliano, Giancarlo Ortaggi, Andrea Masotti ${ }^{*}$ \\ From CMA4CH 2010: Multivariate Analysis and Chemometry to Cultural Heritage and Environment \\ Taormina, Italy. 26-29 September 2010
}

\begin{abstract}
Background: Herbaceous plants are common vegetal species generally exposed, for a limited period of time, to bioavailable environmental pollutants. Heavy metals contamination is the most common form of environmental pollution. Herbaceous plants have never been used as natural bioindicators of environmental pollution, in particular to monitor the amount of heavy metals in soil. In this study, we aimed at assessing the usefulness of using three herbaceous plants (Plantago major L., Taraxacum officinale L. and Urtica dioica L.) and one leguminous (Trifolium pratense L.) as alternative indicators to evaluate soil pollution by heavy metals.

Results: We employed Inductively Coupled Plasma Atomic Emission Spectroscopy (ICP-AES) to assess the concentration of selected heavy metals ( $\mathrm{Cu}, \mathrm{Zn}, \mathrm{Mn}, \mathrm{Pb}, \mathrm{Cr}$ and $\mathrm{Pd}$ ) in soil and plants and we employed statistical analyses to describe the linear correlation between the accumulation of some heavy metals and selected vegetal species. We found that the leaves of Taraxacum officinale $L$. and Trifolium pratense $L$. can accumulate $\mathrm{Cu}$ in a linearly dependent manner with Urtica dioica $L$. representing the vegetal species accumulating the highest fraction of $\mathrm{Pb}$.
\end{abstract}

Conclusions: In this study we demonstrated that common plants can be used as an alternative analytical tool for monitoring selected heavy metals in soil.

\section{Background}

Heavy metals contamination is one of the major kind of environmental pollution in urbanized cities due to emissions from heating, transport, industry and other human activities. In the past, the main contribution to heavy metals contamination has been due to lead used as anti detonating agent in fuels. At the end of 1998, the European Parliament and Council with the Directive 98/ 70/EC prohibited the marketing of leaded petrol within their territory. Since that date, the contribution of lead to heavy metal pollution have to depend from other anthropogenic sources (i.e., exausted batteries, paintings and other industrial wastes). Cadmium, zinc and nickel originate from oils, pneumatics and old car pieces in general, copper from cars and other electric vehicles and manganese prevalently from natural sources. Accumulation

\footnotetext{
* Correspondence: andrea.masotti@uniroma1.it

Chemistry Department, Sapienza University of Rome, P.le A.Moro 5, 00185 Rome, Italy
}

(and distribution) of anthropogenic heavy metals in soil may depend on wet and dry depositions that convey particles from air to soil. Heavy metals may impair plant physiology by reducing respiration and growth, interfering with photosynthetic processes and inhibiting fundamental enzymatic reactions if accumulated at high concentrations. When these toxic metals are present in soil at a low concentration, plants continue to grow uniformly despite accumulating these metals. The ability of plants to accumulate heavy metals into their organs may hence be used to monitor soil pollution, and in particular the amount of heavy metals.

In the past, several authors investigated the distribution of heavy metals in roadside soil [1-4], grass [5] and leaves $[6,7]$ emphasizing lead accumulation in soils and vegetation [8-10], near highways [11], in small mammals [12,13], humans [14] and invertebrates [15,16]. Other authors focused their attention on heavy metals accumulation by 
higher plants in order to study the urban pollution [17-20].

One interesting study on the air pollution by vehicular traffic in Rome was reported [21], but only higher plants have been considered as environmental pollution markers.

In this study, common plants have been considered for two reasons. First, they are ephemeral: they live for a short time and thus they are exposed only for a very specific period of time to bioavailable pollutants. Second, they can be picked up more easily than other higher plants. Therefore, we studied three herbaceous plants (Plantago major, Linnaeus, Taraxacum officinale, Linnaeus and Urtica dioica, Linnaeus) and one leguminous (Trifolium pratense, Linnaeus) and we compared the heavy metals accumulation in roots and leaves. Together with $\mathrm{Cu}, \mathrm{Zn}, \mathrm{Mn}$, and $\mathrm{Pb}$ we decided to consider also $\mathrm{Cr}$ and $\mathrm{Pd}$ to investigate if a significant release from vehicles components or from catalytic converters can occur. Our study is therefore aimed at finding simple and reliable vegetal indicators to monitor environmental pollution and in particular soil pollution by heavy metals.

\section{Experimental \\ Reagents}

Concentrated $\mathrm{HNO}_{3}$ (65\%) was purchased by SigmaAldrich. Standard reference materials (SRM No. 2587 and 2711) were from the National Institute of Standards and Technology, Gaithersburg, USA.

\section{Apparatus}

\section{Analytical determination and data elaboration}

The concentration of selected heavy metals $(\mathrm{Cu}, \mathrm{Mn}, \mathrm{Zn}$, $\mathrm{Pb}, \mathrm{Cr}$ and $\mathrm{Pd}$ ) were determined by means of ICP-AES spectrophotometer (Varian Vista MPX CCD. Simultaneous ICP-OES) equipped with a U5000 AT+ nebulizer (Cetac Technologies). In order to maximize the element sensitivity and to avoid interferences, wavelengths were accurately chosen $(324.754 \mathrm{~nm}$ for $\mathrm{Cu}, 257.610 \mathrm{~nm}$ for Mn, $206.200 \mathrm{~nm}$ for $\mathrm{Zn}, 220.353$ for $\mathrm{Pb}, 267.716$ for $\mathrm{Cr}$ and 340.458 for Pd) and two spectral regions were investigated. To assure a correct calibration of the instrument, at least one standard sample has been run every 10 test samples. Concentrations have been reported as mean values of three replicates. We found that all analytical determinations performed by ICP-MS are affected by an error equal to 5\%. Data and graphics were elaborated with SigmaPlot Ver. 8.0 and Excel.

\section{Methods and procedures}

Soil and plants sampling

For this study we considered four different vegetal species (Plantago major L., Taraxacum officinale L., Urtica dioica $L$. and Trifolium pratense L.) collected in spring (mid-
March), in summer (at the end of June) and in autumn (beginning of October) of year 1999. Five sampling areas (SAs) in the city of Rome have been chosen according to their different level of anthropogenic pollution. In particular, two of these sites (SA1 and SA2) are located close to high-traffic roads (Muro Torto and Olimpica), other two near medium- and low- traffic (SA3 and SA4) roads (Ostiense and Eur) and the last (SA5) from a large park (Pamphili). The latter was assumed as the reference (uncontaminated) site.

Surface soils and plants samples (each weighing about $500 \mathrm{~g}$ ) were taken in triplicate, at the same distance from the street across a $1 \times 1 \mathrm{~m}^{2}$ area by employing a stainless steel trowel to a $20 \mathrm{~cm}$ depth from the surface. After classification, plants and surface soil samples have been put in suitable plastic containers on the same occurrence.

\section{Sample preparation and digestion procedure}

Soil samples coming from the same site were pooled together, air-dried up to dryness, then sieved by passing through a $1 \mathrm{~mm}$ nylon sieve; fractions less than $1 \mathrm{~mm}$ size were further ground in an agate mortar, till all the sample was homogenized. Soil samples (particle size around $0.2 \mathrm{~mm}$ ) were sealed in polyethylene bottles and stored.

The roots and leaves of the collected plants, suitably separated, were repeatedly washed first with tap water then with deionized water and finally air-dried. Roots samples from each of the three plants (of the same species) were pooled together, oven dried $\left(105{ }^{\circ} \mathrm{C}, 48 \mathrm{~h}\right)$ homogenized and grinded in a metal free mill to obtain a fine powder. The same protocol was applied also to leaves.

For analysis, 350-400 mg (exactly weighted) of soil, roots or leaves were digested with $10 \mathrm{ml}$ of concentrated $\mathrm{HNO}_{3}(65 \%)$ for $24 \mathrm{~h}$ at $130{ }^{\circ} \mathrm{C}$ in $25 \mathrm{ml}$ round bottomed flasks equipped with reflux condensers. The vessels were cooled, and stock solutions were obtained by transferring samples in $25 \mathrm{ml}$ volumetric flasks and made up to the mark with deionized water $\left(0.05 \mu \mathrm{Scm}^{-}\right.$ $\left.{ }^{1}\right)$. The solution was filtered through a Whatman 541 paper and stored in glass bottles. Working solutions were obtained by diluting 1:10 (v:v) the correspondent stock solutions. Moreover, we performed also the analysis of blanks (clean mineralization solution) and standard reference materials (SRM) from the National Institute of Standards and Technology, Gaithersburg, USA (SRM No. 2587 and No. 2586 - Trace Elements in Soil containing lead from paint) in the same experimental conditions and by using the same protocol. The recovery varied from 95 to $98 \%$ and all the obtained values $\pm 3 \sigma$ were within the range of certified values. 


\section{Results}

\section{Analytical determinations}

The mean concentration of $\mathrm{Cu}, \mathrm{Mn}, \mathrm{Zn}, \mathrm{Pb}, \mathrm{Cr}$ and $\mathrm{Pd}$ from surface soil, Plantago major L., Taraxacum officinale L., Urtica dioica $L$. and Trifolium pratense L. (both roots and leaves) have been summarized in Tables 1, 2, 3,4 .

\section{Heavy metals in soil}

We found that $\mathrm{Cu}, \mathrm{Mn}, \mathrm{Zn}, \mathrm{Pb}, \mathrm{Cr}$ and $\mathrm{Pd}$ amount in soil varies with the order $\mathrm{SA} 1 \approx \mathrm{SA} 2>\mathrm{SA} 3>\mathrm{SA} 4>\mathrm{SA} 5$, being SA1 the most polluted area and SA5 the less contaminated one. Heavy metals concentration we found, is therefore closely linked to the level of contamination of the different sampling areas. The trend observed is independent on vegetal species considered and/or seasons. In every sampling site, among the heavy metals taken into consideration, $\mathrm{Mn}$ and $\mathrm{Pb}$ are the two most abundant whereas, $\mathrm{Cr}$ and Pd display the lowest concentrations.

The results of the heavy metals determined in soil seems to evidence a seasonal dependence. Fig. 1 reports an indicative example of the seasonal variation of heavy metal concentration for $\mathrm{Cu}$ and $\mathrm{Pb}$ in Plantago major $L$.

Table 1 Heavy metals concentrations in Plantago major L. Cu, Mn, Zn, Pb, $\mathbf{C r}$ and Pd soil, roots and leaves concentrations (ppm) in Plantago major L.

\begin{tabular}{|c|c|c|c|c|c|c|c|c|c|}
\hline \multirow[b]{2}{*}{$\mathrm{Cu}$} & \multicolumn{3}{|c|}{ SPRING } & \multicolumn{3}{|c|}{ SUMMER } & \multicolumn{3}{|c|}{ AUTUMN } \\
\hline & Soil & Roots & Leaves & Soil & Roots & Leaves & Soil & Roots & Leaves \\
\hline SA1 & $111 \pm 5.6$ & $53 \pm 2.7$ & $17 \pm 0.9$ & $199 \pm 10.0$ & $124 \pm 6.2$ & $60 \pm 3$ & $188 \pm 9.4$ & $67 \pm 3.4$ & $37 \pm 1.9$ \\
\hline SA2 & $126 \pm 6.3$ & $62 \pm 3.1$ & $35 \pm 1.8$ & $214 \pm 10.7$ & $57 \pm 2.9$ & $26 \pm 1.3$ & $195 \pm 9.8$ & $104 \pm 5.2$ & $20 \pm 1$ \\
\hline SA3 & $52 \pm 2.6$ & $20 \pm 1$ & $10 \pm 0.5$ & $137 \pm 6.9$ & $93 \pm 4.7$ & $36 \pm 1.8$ & $129 \pm 6.5$ & $90 \pm 4.5$ & $29 \pm 1.5$ \\
\hline SA4 & $39 \pm 2.0$ & $30 \pm 1.5$ & $21 \pm 1.1$ & $93 \pm 4.7$ & $49 \pm 2.5$ & $18 \pm 0.9$ & $74 \pm 3.7$ & $67 \pm 3.4$ & $37 \pm 1.9$ \\
\hline SA5 & $27 \pm 1.4$ & $23 \pm 1.2$ & $20 \pm 1$ & $52 \pm 2.6$ & $39 \pm 2.0$ & $42 \pm 2.1$ & $43 \pm 2.2$ & $50 \pm 2.5$ & $39 \pm 2.0$ \\
\hline $\mathrm{Mn}$ & Soil & Roots & Leaves & Soil & Roots & Leaves & Soil & Roots & Leaves \\
\hline SA1 & $773 \pm 38.7$ & $129 \pm 6.5$ & $51 \pm 2.6$ & $570 \pm 28.5$ & $160 \pm 8$ & $84 \pm 4.2$ & $627 \pm 31.4$ & $62 \pm 3.1$ & $31 \pm 1.6$ \\
\hline SA2 & $730 \pm 36.5$ & $72 \pm 3.6$ & $29 \pm 1.5$ & $509 \pm 25.5$ & $94 \pm 4.7$ & $34 \pm 1.7$ & $534 \pm 26.7$ & $106 \pm 5.3$ & $21 \pm 1.1$ \\
\hline $\mathrm{SA} 3$ & $784 \pm 39.2$ & $62 \pm 3.1$ & $72 \pm 3.6$ & $579 \pm 29.0$ & $147 \pm 7.4$ & $44 \pm 2.2$ & $579 \pm 29.0$ & $36 \pm 1.8$ & $29 \pm 1.5$ \\
\hline SA4 & $820 \pm 41$ & $171 \pm 8.6$ & $92 \pm 4.6$ & $560 \pm 28$ & $130 \pm 6.5$ & $56 \pm 2.8$ & $600 \pm 30$ & $113 \pm 5.7$ & $51 \pm 2.6$ \\
\hline SA5 & $745 \pm 37.3$ & $48 \pm 2.4$ & $33 \pm 1.7$ & $449 \pm 22.5$ & $80 \pm 4$ & $23 \pm 1.2$ & $552 \pm 27.6$ & $45 \pm 2.3$ & $35 \pm 1.8$ \\
\hline $\mathrm{Zn}$ & Soil & Roots & Leaves & Soil & Roots & Leaves & Soil & Roots & Leaves \\
\hline SA1 & $206 \pm 10.3$ & $106 \pm 5.3$ & $57 \pm 2.9$ & $303 \pm 15.2$ & $199 \pm 10.0$ & $95 \pm 4.8$ & $342 \pm 17.1$ & $212 \pm 10.6$ & $121 \pm 6.1$ \\
\hline $\mathrm{SA} 2$ & $226 \pm 11.3$ & $158 \pm 7.9$ & $91 \pm 4.6$ & $321 \pm 16.1$ & $167 \pm 8.4$ & $75 \pm 3.8$ & $334 \pm 16.7$ & $156 \pm 7.8$ & $75 \pm 3.8$ \\
\hline SA3 & $104 \pm 5.2$ & $72 \pm 3.6$ & $51 \pm 2.6$ & $290 \pm 14.5$ & $134 \pm 6.7$ & $76 \pm 3.8$ & $368 \pm 18.4$ & $181 \pm 9.1$ & $95 \pm 4.8$ \\
\hline SA4 & $98 \pm 4.9$ & $75 \pm 3.8$ & $49 \pm 2.5$ & $240 \pm 12$ & $104 \pm 5.2$ & $51 \pm 2.6$ & $290 \pm 14.5$ & $154 \pm 7.7$ & $83 \pm 4.2$ \\
\hline SA5 & $71 \pm 3.6$ & $37 \pm 1.9$ & $39 \pm 2.0$ & $122 \pm 6.1$ & $90 \pm 4.5$ & $61 \pm 3.1$ & $116 \pm 5.8$ & $73 \pm 3.7$ & $68 \pm 3.4$ \\
\hline $\mathrm{Pb}$ & Soil & Roots & Leaves & Soil & Roots & Leaves & Soil & Roots & Leaves \\
\hline SA1 & $578 \pm 28.9$ & $54 \pm 2.7$ & $12 \pm 0.6$ & $840 \pm 42$ & $35 \pm 1.8$ & $11 \pm 0.6$ & $686 \pm 34.3$ & $28 \pm 1.4$ & $8 \pm 0.4$ \\
\hline $\mathrm{SA} 2$ & $488 \pm 24.4$ & $38 \pm 1.9$ & n.d. & $792 \pm 39.6$ & $6 \pm 0.3$ & n.d. & $596 \pm 29.8$ & $15 \pm 0.8$ & $3 \pm 0.2$ \\
\hline SA3 & $276 \pm 13.8$ & n.d. & n.d. & $546 \pm 27.3$ & $26 \pm 1.3$ & n.d. & $523 \pm 26.2$ & $18 \pm 0.9$ & $3 \pm 0.2$ \\
\hline SA4 & $219 \pm 11.0$ & n.d. & n.d. & $425 \pm 21.3$ & $16 \pm 0.8$ & n.d. & $121 \pm 6.1$ & $10 \pm 0.5$ & $4 \pm 0.2$ \\
\hline SA5 & $137 \pm 6.9$ & n.d. & n.d. & $58 \pm 2.9$ & $2 \pm 0.1$ & n.d. & $82 \pm 4.1$ & $4 \pm 0.2$ & n.d. \\
\hline $\mathrm{Cr}$ & Soil & Roots & Leaves & Soil & Roots & Leaves & Soil & Roots & Leaves \\
\hline SA1 & $40 \pm 2$ & $8 \pm 0.4$ & $2 \pm 0.1$ & $24 \pm 1.2$ & $3 \pm 0.2$ & $1 \pm 0.1$ & $26 \pm 1.3$ & $6 \pm 0.3$ & $1 \pm 0.1$ \\
\hline $\mathrm{SA} 2$ & $43 \pm 2.2$ & $9 \pm 0.5$ & $3 \pm 0.2$ & $21 \pm 1.1$ & $3 \pm 0.2$ & $1 \pm 0.1$ & $30 \pm 1.5$ & $3 \pm 0.2$ & n.d. \\
\hline SA3 & $33 \pm 1.7$ & $3 \pm 0.2$ & $1 \pm 0.1$ & $29 \pm 1.5$ & $5 \pm 0.3$ & $2 \pm 0.1$ & $33 \pm 1.7$ & $2 \pm 0.1$ & n.d. \\
\hline SA4 & $35 \pm 1.8$ & $7 \pm 0.4$ & $2 \pm 0.1$ & $27 \pm 1.4$ & $6 \pm 0.3$ & $1 \pm 0.1$ & $22 \pm 1.1$ & $3 \pm 0.2$ & n.d. \\
\hline SA5 & $22 \pm 1.1$ & $3 \pm 0.2$ & $1 \pm 0.1$ & $11 \pm 0.6$ & $4 \pm 0.2$ & n.d. & $16 \pm 0.8$ & $1 \pm 0.1$ & n.d. \\
\hline $\mathrm{Pd}$ & Soil & Roots & Leaves & Soil & Roots & Leaves & Soil & Roots & Leaves \\
\hline SA1 & $71 \pm 3.6$ & $7 \pm 0.4$ & $3 \pm 0.2$ & $74 \pm 3.7$ & $3 \pm 0.2$ & $1 \pm 0.1$ & $72 \pm 3.6$ & $5 \pm 0.3$ & $1 \pm 0.1$ \\
\hline SA2 & $70 \pm 3.5$ & $7 \pm 0.4$ & $2 \pm 0.1$ & $72 \pm 3.6$ & $4 \pm 0.2$ & $2 \pm 0.1$ & $77 \pm 3.9$ & $6 \pm 0.3$ & $1 \pm 0.1$ \\
\hline SA3 & $73 \pm 3.7$ & $4 \pm 0.2$ & $1 \pm 0.1$ & $70 \pm 3.5$ & $4 \pm 0.2$ & $1 \pm 0.1$ & $74 \pm 3.7$ & $5 \pm 0.3$ & $1 \pm 0.1$ \\
\hline SA4 & $67 \pm 3.4$ & $7 \pm 0.4$ & $2 \pm 0.1$ & $73 \pm 3.7$ & $5 \pm 0.3$ & $2 \pm 0.1$ & $67 \pm 3.4$ & $7 \pm 0.4$ & $2 \pm 0.1$ \\
\hline SA5 & $41 \pm 2.1$ & $2 \pm 0.1$ & $1 \pm 0.1$ & $41 \pm 2.1$ & $3 \pm 0.2$ & $1 \pm 0.1$ & $44 \pm 2.2$ & $3 \pm 0.2$ & $1 \pm 0.1$ \\
\hline
\end{tabular}


Table 2 Heavy metals concentrations in Taraxacum officinale L. $\mathbf{C u}, \mathbf{M n}, \mathbf{Z n}, \mathbf{P b}, \mathbf{C r}$ and Pd soil, roots and leaves concentrations (ppm) in Taraxacum officinale $L$.

\begin{tabular}{|c|c|c|c|c|c|c|c|c|c|}
\hline & & SPRING & & & SUMMER & & & AUTUMN & \\
\hline $\mathrm{Cu}$ & Soil & Roots & Leaves & Soil & Roots & Leaves & Soil & Roots & Leaves \\
\hline SA1 & $126 \pm 6.3$ & $36 \pm 1.8$ & $35 \pm 1.8$ & $136 \pm 6.8$ & $95 \pm 4.8$ & $51 \pm 2.6$ & $131 \pm 6.6$ & $97 \pm 4.9$ & $52 \pm 2.6$ \\
\hline $\mathrm{SA} 2$ & $116 \pm 5.8$ & $46 \pm 2.3$ & $39 \pm 2.0$ & $144 \pm 7.2$ & $64 \pm 3.2$ & $42 \pm 2.1$ & $142 \pm 7.1$ & $71 \pm 3.6$ & $45 \pm 2.3$ \\
\hline SA3 & $110 \pm 5.5$ & $31 \pm 1.6$ & $37 \pm 1.9$ & $155 \pm 7.8$ & $55 \pm 2.8$ & $39 \pm 2.0$ & $99 \pm 5.0$ & $27 \pm 1.4$ & $34 \pm 1.7$ \\
\hline SA4 & $54 \pm 2.7$ & $40 \pm 2$ & $23 \pm 1.2$ & $127 \pm 6.4$ & $31 \pm 1.6$ & $26 \pm 1.3$ & $74 \pm 3.7$ & $30 \pm 1.5$ & $24 \pm 1.2$ \\
\hline SA5 & $32 \pm 1.6$ & $15 \pm 0.8$ & $15 \pm 0.8$ & $104 \pm 5.2$ & $31 \pm 1.6$ & $24 \pm 1.2$ & $55 \pm 2.8$ & $25 \pm 1.3$ & $10 \pm 0.5$ \\
\hline $\mathrm{Mn}$ & Soil & Roots & Leaves & Soil & Roots & Leaves & Soil & Roots & Leaves \\
\hline SA1 & $809 \pm 40.5$ & $49 \pm 2.5$ & $39 \pm 2.0$ & $546 \pm 27.3$ & $61 \pm 3.1$ & $41 \pm 2.1$ & $624 \pm 31.2$ & $43 \pm 2.2$ & $19 \pm 1.0$ \\
\hline SA2 & $720 \pm 36$ & $115 \pm 5.8$ & $64 \pm 3.2$ & $576 \pm 28.8$ & $112 \pm 5.6$ & $35 \pm 1.8$ & $602 \pm 30.1$ & $61 \pm 3.1$ & $42 \pm 2.1$ \\
\hline SAB & $755 \pm 37.8$ & $76 \pm 3.8$ & $74 \pm 3.7$ & $571 \pm 28.6$ & $83 \pm 4.2$ & $58 \pm 2.9$ & $583 \pm 29.2$ & $65 \pm 3.3$ & $38 \pm 1.9$ \\
\hline SA4 & $788 \pm 39.4$ & $91 \pm 4.6$ & $98 \pm 4.9$ & $600 \pm 30$ & $66 \pm 3.3$ & $51 \pm 2.6$ & $580 \pm 29$ & $104 \pm 5.2$ & $54 \pm 2.7$ \\
\hline SA5 & $646 \pm 32.3$ & $45 \pm 2.3$ & $44 \pm 2.2$ & $631 \pm 31.6$ & $86 \pm 4.3$ & $59 \pm 3.0$ & $624 \pm 31.2$ & $59 \pm 3.0$ & $32 \pm 1.6$ \\
\hline $\mathrm{Zn}$ & Soil & Roots & Leaves & Soil & Roots & Leaves & Soil & Roots & Leaves \\
\hline SA1 & $229 \pm 11.5$ & $155 \pm 7.8$ & $133 \pm 6.7$ & $374 \pm 18.7$ & $211 \pm 10.6$ & $148 \pm 7.4$ & $742 \pm 37.1$ & $227 \pm 11.4$ & $90 \pm 4.5$ \\
\hline SA2 & $220 \pm 11$ & $157 \pm 7.9$ & $121 \pm 6.1$ & $426 \pm 21.3$ & $234 \pm 11.7$ & $150 \pm 7.5$ & $678 \pm 33.9$ & $265 \pm 13.3$ & $137 \pm 6$ \\
\hline SA3 & $215 \pm 10.8$ & $119 \pm 6.0$ & $109 \pm 5.5$ & $263 \pm 13.2$ & $152 \pm 7.6$ & $105 \pm 5.3$ & $694 \pm 34.7$ & $187 \pm 9.4$ & $64 \pm 3.2$ \\
\hline SA4 & $101 \pm 5.1$ & $94 \pm 4.7$ & $79 \pm 4.0$ & $254 \pm 12.7$ & $72 \pm 3.6$ & $80 \pm 4$ & $393 \pm 19.7$ & $73 \pm 3.7$ & $55 \pm 2.8$ \\
\hline SA5 & $61 \pm 3.1$ & $59 \pm 3.0$ & $70 \pm 3.5$ & $93 \pm 4.7$ & $70 \pm 3.5$ & $80 \pm 4$ & $140 \pm 7$ & $73 \pm 3.7$ & $40 \pm 2$ \\
\hline $\mathrm{Pb}$ & Soil & Roots & Leaves & Soil & Roots & Leaves & Soil & Roots & Leaves \\
\hline SA1 & $627 \pm 31.4$ & $68 \pm 3.4$ & $8 \pm 0.4$ & $796 \pm 39.8$ & $109 \pm 5.5$ & $22 \pm 1.1$ & $730 \pm 36.5$ & $84 \pm 4.2$ & $11 \pm 0.6$ \\
\hline SA2 & $588 \pm 29.4$ & $75 \pm 3.8$ & n.d. & $769 \pm 38.5$ & $155 \pm 7.8$ & $28 \pm 1.4$ & $730 \pm 36.5$ & $108 \pm 5.4$ & $22 \pm 1.1$ \\
\hline SAB & $206 \pm 10.3$ & n.d. & n.d. & $644 \pm 32.2$ & $26 \pm 1.3$ & $12 \pm 0.6$ & $560 \pm 28$ & $15 \pm 0.8$ & $3 \pm 0.2$ \\
\hline SA4 & $244 \pm 12.2$ & n.d. & n.d. & $371 \pm 18.6$ & $11 \pm 0.6$ & $8 \pm 0.4$ & $107 \pm 5.4$ & $9 \pm 0.5$ & $2 \pm 0.1$ \\
\hline SA5 & $148 \pm 7.4$ & n.d. & n.d. & $174 \pm 8.7$ & $6 \pm 0.3$ & $4 \pm 0.2$ & $89 \pm 4.5$ & $4 \pm 0.2$ & $1 \pm 0.1$ \\
\hline $\mathrm{Cr}$ & Soil & Roots & Leaves & Soil & Roots & Leaves & Soil & Roots & Leaves \\
\hline SA1 & $35 \pm 1.8$ & $7 \pm 0.4$ & $3 \pm 0.2$ & $29 \pm 1.5$ & $8 \pm 0.4$ & $2 \pm 0.1$ & $30 \pm 1.5$ & $3 \pm 0.2$ & $1 \pm 0.1$ \\
\hline $\mathrm{SA} 2$ & $38 \pm 1.9$ & $10 \pm 0.5$ & $5 \pm 0.3$ & $34 \pm 1.7$ & $10 \pm 0.5$ & $3 \pm 0.2$ & $35 \pm 1.8$ & $4 \pm 0.2$ & $1 \pm 0.1$ \\
\hline SA3 & $40 \pm 2$ & $4 \pm 0.2$ & $3 \pm 0.2$ & $31 \pm 1.6$ & $7 \pm 0.4$ & $1 \pm 0.1$ & $32 \pm 1.6$ & $3 \pm 0.2$ & $1 \pm 0.1$ \\
\hline SA4 & $45 \pm 2.3$ & $7 \pm 0.4$ & $3 \pm 0.2$ & $29 \pm 1.5$ & $4 \pm 0.2$ & $2 \pm 0.1$ & $21 \pm 1.1$ & n.d. & n.d. \\
\hline SA5 & $33 \pm 1.7$ & $3 \pm 0.2$ & $3 \pm 0.2$ & $28 \pm 1.4$ & $2 \pm 0.1$ & $1 \pm 0.1$ & $28 \pm 1.4$ & n.d. & n.d. \\
\hline
\end{tabular}

Concentrations of $\mathrm{Cu}$ and $\mathrm{Pb}$ reach the maximum value during summer while $\mathrm{Mn}$ reaches the minimum value. $\mathrm{Zn}$ concentration increases from spring to autumn while $\mathrm{Cr}$ and $\mathrm{Pd}$ concentrations remain relatively constant. Other factors can influence the local concentration of heavy metals in soil: temperature, rainfall, evapotranspiration, soil $\mathrm{pH}$ and redox potential. To correlate heavy metals concentration with the level of precipitation, we collected the rainfall data for the city of Rome from the Meteorological Centre of Rome. Superimposing the precipitations records with heavy metals concentrations we were able to observe some characteristic trends. In particular, during spring and autumn when the first and the third sampling occurred, moderate to abundant precipitation were registered whilst in summer rains are rare. The higher temperature and reduced rainfall may hence favour the water evaporation in soils leading to a higher accumulation of metals with respect to spring or autumn. $\mathrm{Cu}$, and $\mathrm{Pb}$ seem to follow such a behaviour, with a maximum concentration during summer (214 ppm and $1266 \mathrm{ppm}$, respectively), while $\mathrm{Zn}$ concentration reaches a maximum during autumn (742 ppm). On the contrary, Mn follows the opposite trend showing the lowest value during summer (449 ppm). Cr and Pd seem not to be influenced by atmospheric conditions and their concentration remain relatively low and constant all over the year (between 15 and 45 ppm for $\mathrm{Cr}$ and between 37 and $77 \mathrm{ppm}$ for $\mathrm{Pd}$ ).

\section{Heavy metals in plants}

Heavy metals found in roots and leaves of the three herbaceous plants (Plantago major L., Taraxacum officinale L. and Urtica dioica L.) and the leguminous Trifolium pratense L., allowed us to conclude that the content of heavy metals in roots is higher than in leaves and that accumulation process of herbaceous plants does not significantly 
Table 3 Heavy metals concentrations in Urtica dioica $\mathrm{L} . \mathrm{Cu}, \mathrm{Mn}, \mathrm{Zn}, \mathrm{Pb}, \mathrm{Cr}$ and $\mathrm{Pd}$ soil, roots and leaves concentrations (ppm) in Urtica dioica $L$.

\begin{tabular}{|c|c|c|c|c|c|c|c|c|c|}
\hline & & SPRING & & & SUMMER & & & AUTUMN & \\
\hline $\mathrm{Cu}$ & Soil & Roots & Leaves & Soil & Roots & Leaves & Soil & Roots & Leaves \\
\hline SA1 & $104 \pm 5.2$ & $42 \pm 2.1$ & $21 \pm 1.1$ & $186 \pm 9.3$ & $106 \pm 5.3$ & $41 \pm 2.1$ & $162 \pm 8.1$ & $51 \pm 2.6$ & $28 \pm 1.4$ \\
\hline SA2 & $92.5 \pm 4.7$ & $22 \pm 1.1$ & $19 \pm 1.0$ & $156 \pm 7.8$ & $100 \pm 5$ & $34 \pm 1.7$ & $169 \pm 8.5$ & $55 \pm 2.8$ & $39 \pm 2.0$ \\
\hline SA3 & $85 \pm 4.3$ & $18 \pm 0.9$ & $14 \pm 0.7$ & $105 \pm 5.3$ & $40 \pm 2$ & $15 \pm 0.8$ & $117 \pm 5.9$ & $61 \pm 3.1$ & $33 \pm 1.7$ \\
\hline SA4 & $38 \pm 1.9$ & $15 \pm 0.8$ & $17 \pm 0.9$ & $101 \pm 5.1$ & $56 \pm 2.8$ & $18 \pm 0.9$ & $105 \pm 5.3$ & $46 \pm 2.3$ & $23 \pm 1.2$ \\
\hline SA5 & $26 \pm 1.3$ & $17 \pm 0.9$ & $13 \pm 0.7$ & $47 \pm 2.4$ & $30 \pm 1.5$ & $20 \pm 1$ & $87 \pm 4.4$ & $51 \pm 2.6$ & $30 \pm 1.5$ \\
\hline $\mathrm{Mn}$ & Soil & Roots & Leaves & Soil & Roots & Leaves & Soil & Roots & Leaves \\
\hline SA1 & $651 \pm 32.6$ & $158 \pm 7.9$ & $41 \pm 2.1$ & $580 \pm 29$ & $147 \pm 7.4$ & $71 \pm 3.6$ & $626 \pm 31.3$ & $163 \pm 8.2$ & $39 \pm 2.0$ \\
\hline $\mathrm{SA} 2$ & $603 \pm 30.2$ & $182 \pm 9.1$ & $37 \pm 1.9$ & $514 \pm 25.7$ & $104 \pm 5.2$ & $29 \pm 1.5$ & $554 \pm 27.7$ & $130 \pm 6.5$ & $34 \pm 1.7$ \\
\hline $\mathrm{SA} 3$ & $626 \pm 31.3$ & $121 \pm 6.1$ & $70 \pm 3.5$ & $532 \pm 26.6$ & $110 \pm 5.5$ & $63 \pm 3.2$ & $640 \pm 32$ & $169 \pm 8.5$ & $48 \pm 2.4$ \\
\hline SA4 & $587 \pm 29.4$ & $111 \pm 5.6$ & $54 \pm 2.7$ & $496 \pm 24.8$ & $86 \pm 4.3$ & $77 \pm 3.9$ & $620 \pm 31$ & $160 \pm 8$ & $78 \pm 3.9$ \\
\hline SA5 & $553 \pm 27.7$ & $98 \pm 4.9$ & $31 \pm 1.6$ & $518 \pm 25.9$ & $121 \pm 6.1$ & $69 \pm 3.5$ & $620 \pm 31$ & $142 \pm 7.1$ & $55 \pm 2.8$ \\
\hline $\mathrm{Zn}$ & Soil & Roots & Leaves & Soil & Roots & Leaves & Soil & Roots & Leaves \\
\hline SA1 & $185 \pm 9.3$ & $97 \pm 4.9$ & $46 \pm 2.3$ & $255 \pm 12.8$ & $144 \pm 7.2$ & $128 \pm 6.4$ & $336 \pm 16.8$ & $198 \pm 9.9$ & $124 \pm 6.2$ \\
\hline $\mathrm{SA} 2$ & $137 \pm 6.9$ & $34 \pm 1.7$ & $19 \pm 1.0$ & $224 \pm 11.2$ & $103 \pm 5.2$ & $100 \pm 5$ & $428 \pm 21.4$ & $210 \pm 10.5$ & $152 \pm 7.6$ \\
\hline SA3 & $152 \pm 7.6$ & $49 \pm 2.5$ & $36 \pm 1.8$ & $150 \pm 7.5$ & $75 \pm 3.8$ & $65 \pm 3.3$ & $374 \pm 18.7$ & $172 \pm 8.6$ & $130 \pm 6.5$ \\
\hline SA4 & $160 \pm 8$ & $58 \pm 2.9$ & $41 \pm 2.1$ & $185 \pm 9.3$ & $91 \pm 4.6$ & $69 \pm 3.5$ & $223 \pm 11.2$ & $130 \pm 6.5$ & $98 \pm 4.9$ \\
\hline SA5 & $92 \pm 4.6$ & $26 \pm 1.3$ & $14 \pm 0.7$ & $147 \pm 7.4$ & $112 \pm 5.6$ & $95 \pm 4.8$ & $204 \pm 10.2$ & $145 \pm 7.3$ & $120 \pm 6$ \\
\hline $\mathrm{Pb}$ & Soil & Roots & Leaves & Soil & Roots & Leaves & Soil & Roots & Leaves \\
\hline SA1 & $528 \pm 26.4$ & $75 \pm 3.8$ & $21 \pm 1.1$ & $888 \pm 44.4$ & $81 \pm 4.1$ & $23 \pm 1.2$ & $710 \pm 35.5$ & $95 \pm 4.8$ & $30 \pm 1.5$ \\
\hline $\mathrm{SA} 2$ & $452 \pm 22.6$ & $73 \pm 3.7$ & $19 \pm 1.0$ & $971 \pm 48.6$ & $60 \pm 3$ & $21 \pm 1.1$ & $854 \pm 42.7$ & $85 \pm 4.3$ & $23 \pm 1.2$ \\
\hline SA3 & $215 \pm 10.8$ & $44 \pm 2.2$ & $13 \pm 0.7$ & $548 \pm 27.4$ & $46 \pm 2.3$ & $14 \pm 0.7$ & $434 \pm 21.7$ & $59 \pm 3.0$ & $16 \pm 0.8$ \\
\hline SA4 & $152 \pm 7.6$ & $23 \pm 1.2$ & $11 \pm 0.6$ & $294 \pm 14.7$ & $37 \pm 1.9$ & $13 \pm 0.7$ & $230 \pm 11.5$ & $43 \pm 2.2$ & $14 \pm 0.7$ \\
\hline SA5 & $136 \pm 6.8$ & $32 \pm 1.6$ & $9 \pm 0.5$ & $202 \pm 10.1$ & $34 \pm 1.7$ & $10 \pm 0.5$ & $198 \pm 9.9$ & $37 \pm 1.9$ & $12 \pm 0.6$ \\
\hline $\mathrm{Cr}$ & Soil & Roots & Leaves & Soil & Roots & Leaves & Soil & Roots & Leaves \\
\hline SA1 & $28 \pm 1.4$ & $5 \pm 0.3$ & $3 \pm 0.2$ & $36 \pm 1.8$ & $10 \pm 0.5$ & $5 \pm 0.3$ & $28 \pm 1.4$ & $6 \pm 0.3$ & $2 \pm 0.1$ \\
\hline SA2 & $26 \pm 1.3$ & $7 \pm 0.4$ & $4 \pm 0.2$ & $40 \pm 2$ & $12 \pm 0.6$ & $4 \pm 0.2$ & $30 \pm 1.5$ & $7 \pm 0.4$ & $3 \pm 0.2$ \\
\hline SA3 & $22 \pm 1.1$ & $5 \pm 0.3$ & $3 \pm 0.2$ & $43 \pm 2.2$ & $10 \pm 0.5$ & $4 \pm 0.2$ & $29 \pm 1.5$ & $4 \pm 0.2$ & $2 \pm 0.1$ \\
\hline SA4 & $21 \pm 1.1$ & $7 \pm 0.4$ & $5 \pm 0.3$ & $41 \pm 2.1$ & $11 \pm 0.6$ & $4 \pm 0.2$ & $27 \pm 1.4$ & $5 \pm 0.3$ & $2 \pm 0.1$ \\
\hline SA5 & $23 \pm 1.2$ & $5 \pm 0.3$ & $2 \pm 0.1$ & $25 \pm 1.3$ & $7 \pm 0.4$ & $3 \pm 0.2$ & $21 \pm 1.1$ & $4 \pm 0.2$ & $1 \pm 0.1$ \\
\hline $\mathrm{Pd}$ & Soil & Roots & Leaves & Soil & Roots & Leaves & Soil & Roots & Leaves \\
\hline SA1 & $66 \pm 3.3$ & $12 \pm 0.6$ & $5 \pm 0.3$ & $65 \pm 3.3$ & $9 \pm 0.5$ & $3 \pm 0.2$ & $68 \pm 3.4$ & $13 \pm 0.7$ & $3 \pm 0.2$ \\
\hline SA2 & $71 \pm 3.6$ & $16 \pm 0.8$ & $9 \pm 0.5$ & $72 \pm 3.6$ & $10 \pm 0.5$ & $3 \pm 0.2$ & $72 \pm 3.6$ & $13 \pm 0.7$ & $3 \pm 0.2$ \\
\hline SA3 & $76 \pm 3.8$ & $16 \pm 0.8$ & $7 \pm 0.4$ & $70 \pm 3.5$ & $10 \pm 0.5$ & $2 \pm 0.1$ & $65 \pm 3.3$ & $10 \pm 0.5$ & $2 \pm 0.1$ \\
\hline SA4 & $67 \pm 3.4$ & $18 \pm 0.9$ & $9 \pm 0.5$ & $77 \pm 3.9$ & $11 \pm 0.6$ & $3 \pm 0.2$ & $70 \pm 3.5$ & $13 \pm 0.7$ & $3 \pm 0.2$ \\
\hline SA5 & $55 \pm 2.8$ & $8 \pm 0.4$ & $4 \pm 0.2$ & $57 \pm 2.9$ & $5 \pm 0.3$ & $1 \pm 0.1$ & $59 \pm 3.0$ & $6 \pm 0.3$ & $1 \pm 0.1$ \\
\hline
\end{tabular}

differ from that of leguminous plants: the higher the metal concentration in the soil, the higher the concentration in roots and consequently in leaves (Fig. 2).

We further analyzed the correlation between heavy metals content in soil and in leaves of the various vegetal species. We calculated the mean value of heavy metals concentrations in soil and leaves taking into account the values obtained in the three seasons. In this calculation we also considered all the sampling areas in order to analyze different levels of pollution. We calculated the correlation coefficients (Pearson's correlation) between these two set of data and we considered only those metals with $r \geq 0.95$. We therefore found that for Plantago major L. Mn has a correlation coefficient of 0.950, in Taraxacum officinale L. Cu has a coefficient of 0.984, in Urtica dioica $L$. Pb has a correlation of 0.952 while in Trifolium pratense $L$. $\mathrm{Cu}$ and $\mathrm{Pb}$ have coefficients of 0.956 and 0.962 , respectively (Table 5 and Fig. 3).

\section{Discussion}

\section{Heavy metals in soil}

The amount of heavy metals in soil is extremely variable and these differences are more clearly emphasized if we 
Table 4 Heavy metals concentrations in Trifolium pratense L. $\mathbf{C u}, \mathbf{M n}, \mathbf{Z n}, \mathbf{P b}, \mathbf{C r}$ and $\mathbf{P d}$ soil, roots and leaves concentrations (ppm) in Trifolium pratense $L$.

\begin{tabular}{|c|c|c|c|c|c|c|c|c|c|}
\hline & & SPRING & & & SUMMER & & & AUTUMN & \\
\hline $\mathrm{Cu}$ & Soil & Roots & Leaves & Soil & Roots & Leaves & Soil & Roots & Leaves \\
\hline SA1 & $93.1 \pm 4.7$ & $25.7 \pm 1.3$ & $20.5 \pm 1.0$ & $160.2 \pm 8.0$ & $103.3 \pm 5.2$ & $82.1 \pm 4.1$ & $126.4 \pm 6.3$ & $57.2 \pm 2.9$ & $26.5 \pm 1.3$ \\
\hline SA2 & $66.2 \pm 3.3$ & $21.3 \pm 1.1$ & $20.1 \pm 1.0$ & $132.5 \pm 6.6$ & $79.2 \pm 4.0$ & $57.8 \pm 2.9$ & $108.6 \pm 5.4$ & $80.1 \pm 4.0$ & $35.1 \pm 1.8$ \\
\hline SA3 & $55.1 \pm 2.8$ & $16.8 \pm 0.8$ & $10.3 \pm 0.5$ & $82.3 \pm 4.1$ & $31.5 \pm 1.6$ & $16.2 \pm 0.8$ & $65.6 \pm 3.3$ & $44.3 \pm 2.2$ & $23.5 \pm 1.2$ \\
\hline SA4 & $40.3 \pm 2.0$ & $21.5 \pm 1.1$ & $16.3 \pm 0.8$ & $97.7 \pm 4.9$ & $50.6 \pm 2.5$ & $27.3 \pm 1.4$ & $79 \pm 4.0$ & $46.5 \pm 2.3$ & $25.6 \pm 1.3$ \\
\hline SA5 & $36.1 \pm 1.8$ & $21.3 \pm 1.1$ & $11.2 \pm 0.6$ & $41.5 \pm 2.1$ & $35.4 \pm 1.8$ & $18.3 \pm 0.9$ & $49.3 \pm 2.5$ & $33.1 \pm 1.7$ & $19.8 \pm 1.0$ \\
\hline $\mathrm{Mn}$ & Soil & Roots & Leaves & Soil & Roots & Leaves & Soil & Roots & Leaves \\
\hline SA1 & $640 \pm 32$ & $147.2 \pm 7.4$ & $39.3 \pm 2.0$ & $592 \pm 29.6$ & $166.3 \pm 8.3$ & $45.2 \pm 2.3$ & $597 \pm 29.85$ & $34 \pm 1.7$ & $63 \pm 3.2$ \\
\hline SA2 & $581 \pm 29.1$ & $104.5 \pm 5.2$ & $27.1 \pm 1.4$ & $527 \pm 26.4$ & $113.5 \pm 5.7$ & $37.8 \pm 1.9$ & $569 \pm 28.5$ & $115 \pm 5.8$ & $50 \pm 2.5$ \\
\hline SA3 & $608 \pm 30.4$ & $115.1 \pm 5.8$ & $32.1 \pm 1.6$ & $560 \pm 28$ & $128.7 \pm 6.4$ & $36.5 \pm 1.8$ & $600 \pm 30$ & $92 \pm 4.6$ & $78 \pm 3.9$ \\
\hline SA4 & $558 \pm 27.9$ & $100.2 \pm 5.0$ & $24.2 \pm 1.2$ & $503 \pm 25.2$ & $124.2 \pm 6.2$ & $31.2 \pm 1.6$ & $583 \pm 29.1$ & $67 \pm 3.4$ & $57 \pm 2.9$ \\
\hline SA5 & $510.7 \pm 25.5$ & $96.3 \pm 4.8$ & $21.8 \pm 1.09$ & $467.2 \pm 23.4$ & $114.1 \pm 5.7$ & $25.1 \pm 1.3$ & $548 \pm 27.4$ & $43 \pm 2.2$ & $61 \pm 3.1$ \\
\hline $\mathrm{Zn}$ & Soil & Roots & Leaves & Soil & Roots & Leaves & Soil & Roots & Leaves \\
\hline SA1 & $251 \pm 12.6$ & $82.5 \pm 4.1$ & $43.2 \pm 2.2$ & $369 \pm 18.5$ & $130.2 \pm 6.5$ & $50.5 \pm 2.5$ & $407.2 \pm 20.4$ & $232 \pm 11.6$ & $141 \pm 7.1$ \\
\hline $\mathrm{SA} 2$ & $168.3 \pm 8.4$ & $79.2 \pm 4.0$ & $44.1 \pm 2.2$ & $336 \pm 16.8$ & $101.5 \pm 5.1$ & $49.1 \pm 2.5$ & $492.2 \pm 24.6$ & $289 \pm 14.5$ & $127 \pm 6.4$ \\
\hline SA3 & $188.3 \pm 9.4$ & $60.2 \pm 3.0$ & $37.1 \pm 1.9$ & $306 \pm 15.3$ & $99.2 \pm 5.0$ & $49.1 \pm 2.5$ & $461.2 \pm 23.1$ & $282 \pm 14.1$ & $91 \pm 4.6$ \\
\hline SA4 & $196.2 \pm 9.8$ & $61.3 \pm 3.1$ & $36.8 \pm 1.8$ & $290 \pm 14.5$ & $96.5 \pm 4.8$ & $41.2 \pm 2.1$ & $425.3 \pm 21.265$ & $94 \pm 4.7$ & $85 \pm 4.3$ \\
\hline SA5 & $126.5 \pm 6.3$ & $38.4 \pm 1.9$ & $25.6 \pm 1.3$ & $201.2 \pm 10.1$ & $85.2 \pm 4.3$ & $30.2 \pm 1.5$ & $255.1 \pm 12.8$ & $81 \pm 4.1$ & $73 \pm 3.7$ \\
\hline $\mathrm{Pb}$ & Soil & Roots & Leaves & Soil & Roots & Leaves & Soil & Roots & Leaves \\
\hline SA1 & $698 \pm 34.9$ & $7 \pm 0.35$ & $15 \pm 0.8$ & $1163 \pm 58.2$ & $48 \pm 2.4$ & $6 \pm 0.3$ & $1051 \pm 52.6$ & $32 \pm 1.6$ & $12 \pm 0.6$ \\
\hline SA2 & $624 \pm 31.2$ & $0 \pm 0$ & $5 \pm 0.3$ & $1266 \pm 63.3$ & $58 \pm 2.9$ & $12 \pm 0.6$ & $1080 \pm 54$ & $28 \pm 1.4$ & $14 \pm 0.7$ \\
\hline SA3 & $236 \pm 11.8$ & $0 \pm 0$ & $11 \pm 0.6$ & $529 \pm 26.5$ & $9 \pm 0.5$ & $4 \pm 0.2$ & $490 \pm 24.5$ & $4 \pm 0.2$ & $9 \pm 0.5$ \\
\hline SA4 & $159 \pm 8.0$ & $0 \pm 0$ & $0 \pm 0$ & $349 \pm 17.5$ & $30 \pm 1.5$ & $4 \pm 0.2$ & $106 \pm 5.3$ & $10 \pm 0.5$ & $4 \pm 0.2$ \\
\hline SA5 & $126 \pm 6.3$ & $4 \pm 0.2$ & $0 \pm 0$ & $110 \pm 5.5$ & $10 \pm 0.5$ & $6 \pm 0.3$ & $80 \pm 4$ & $2 \pm 0.1$ & $2 \pm 0.1$ \\
\hline $\mathrm{Cr}$ & Soil & Roots & Leaves & Soil & Roots & Leaves & Soil & Roots & Leaves \\
\hline SA1 & $25 \pm 1.3$ & $2 \pm 0.1$ & $0 \pm 0$ & $31 \pm 1.6$ & $3 \pm 0.2$ & $1 \pm 0.1$ & $30 \pm 1.5$ & $3 \pm 0.2$ & $3 \pm 0.2$ \\
\hline SA2 & $22 \pm 1.1$ & $4 \pm 0.2$ & $2 \pm 0.1$ & $37 \pm 1.9$ & $4 \pm 0.2$ & $2 \pm 0.1$ & $31 \pm 1.6$ & $3 \pm 0.2$ & $1 \pm 0.1$ \\
\hline SA3 & $26 \pm 1.3$ & $0 \pm 0$ & $1 \pm 0.1$ & $35 \pm 1.8$ & $0 \pm 0$ & $0 \pm 0$ & $25 \pm 1.3$ & $2 \pm 0.1$ & $1 \pm 0.1$ \\
\hline SA4 & $29 \pm 1.5$ & $3 \pm 0.2$ & $1 \pm 0.1$ & $31 \pm 1.6$ & $3 \pm 0.2$ & $2 \pm 0.1$ & $21 \pm 1.1$ & $2 \pm 0.1$ & $1 \pm 0.1$ \\
\hline SA5 & $22 \pm 1.1$ & $0 \pm 0$ & $2 \pm 0.1$ & $23 \pm 1.2$ & $0 \pm 0$ & $0 \pm 0$ & $15 \pm 0.8$ & $2 \pm 0.1$ & $1 \pm 0.1$ \\
\hline Pd & Soil & Roots & Leaves & Soil & Roots & Leaves & Soil & Roots & Leaves \\
\hline SA1 & $70 \pm 3.5$ & $5 \pm 0.3$ & $0 \pm 0$ & $71 \pm 3.6$ & $5 \pm 0.3$ & $2 \pm 0.1$ & $68 \pm 3.4$ & $5 \pm 0.3$ & $2 \pm 0.1$ \\
\hline SA2 & $67 \pm 3.4$ & $4 \pm 0.2$ & $2 \pm 0.1$ & $68 \pm 3.4$ & $4 \pm 0.2$ & $2 \pm 0.1$ & $66 \pm 3.3$ & $4 \pm 0.2$ & $2 \pm 0.1$ \\
\hline SA3 & $69 \pm 3.5$ & $5 \pm 0.3$ & $0 \pm 0$ & $67 \pm 3.4$ & $4 \pm 0.2$ & $1 \pm 0.1$ & $60 \pm 3$ & $5 \pm 0.3$ & $1 \pm 0.1$ \\
\hline SA4 & $69 \pm 3.5$ & $5 \pm 0.3$ & $2 \pm 0.1$ & $60 \pm 3$ & $3 \pm 0.2$ & $1 \pm 0.1$ & $55 \pm 2.8$ & $4 \pm 0.2$ & $2 \pm 0.1$ \\
\hline SA5 & $37 \pm 1.9$ & $1 \pm 0.1$ & $0 \pm 0$ & $45 \pm 2.3$ & $2 \pm 0.1$ & $1 \pm 0.1$ & $47 \pm 2.4$ & $2 \pm 0.1$ & $1 \pm 0.1$ \\
\hline
\end{tabular}

consider different sampling areas. Different anthropogenic activities may locally alter the amount of some heavy metals, especially of those sites located near hightraffic roads. We found that the amount of some of these metals can be very high (higher than 1000 ppm for some metals) while in the control site (a non polluted park) the concentrations are relatively low. In this study we did not evaluate the effect of the various vegetal species in determining a different 'local environment' that we selected for analytical determination. We did not considered also the various effects of $\mathrm{pH}$, temperature and other physicochemical parameters that can influence the relative heavy metals concentration. However, we found that heavy metals concentration directly correlate with the degree of pollution and, as a consequence, of anthropogenic activity in agreement with previous authors that reported that the principal source of heavy metals pollution (96\% for $\mathrm{Pb}, 66 \%$ for $\mathrm{Zn}$ and $56 \%$ for $\mathrm{Cu}$ ) originates from human activities [22].

\section{Seasonal variation of heavy metals in soil}

We found a seasonal variation of heavy metals concentration in soil, that we ascribed to a different level of metal dissolution due to rainfall. In fact, during summer 


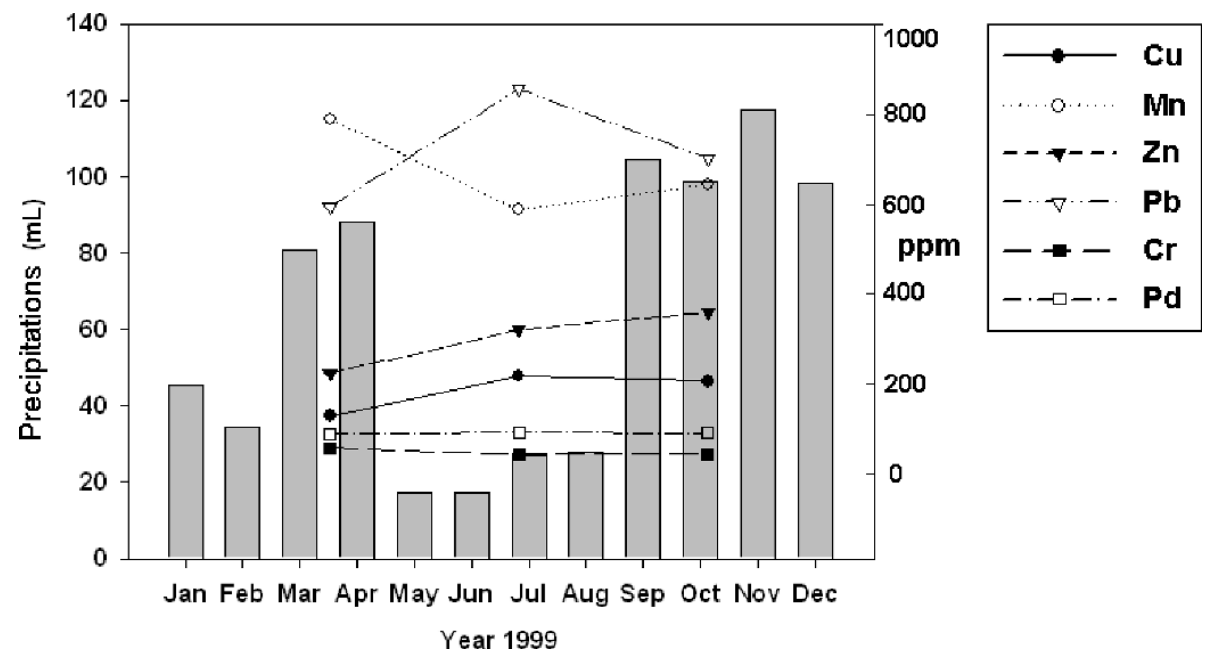

Figure 1 Behaviour of heavy metals concentrations as a function of seasonal precipitations. $\mathrm{Cu}, \mathrm{Mn}, \mathrm{Zn}, \mathrm{Pb}, \mathrm{Cr}$ and $\mathrm{Pd}$ concentrations (ppm) in spring, summer and autumn as a function of precipitations in Rome (Year 1999). As indicative example, metal concentrations were reported as mean values found in SA1-SA5 soils in Plantago major $L$.

the rainfalls are reduced if compared to spring or autumn and high temperatures (or an increase in evapotranspiration) favour an increase of metals concentrations.
Manganese has been found almost equally distributed in all the sampling areas and this indicates that the presence of this metal in soil was not only due to anthropogenic sources (as in most polluted areas) but also to

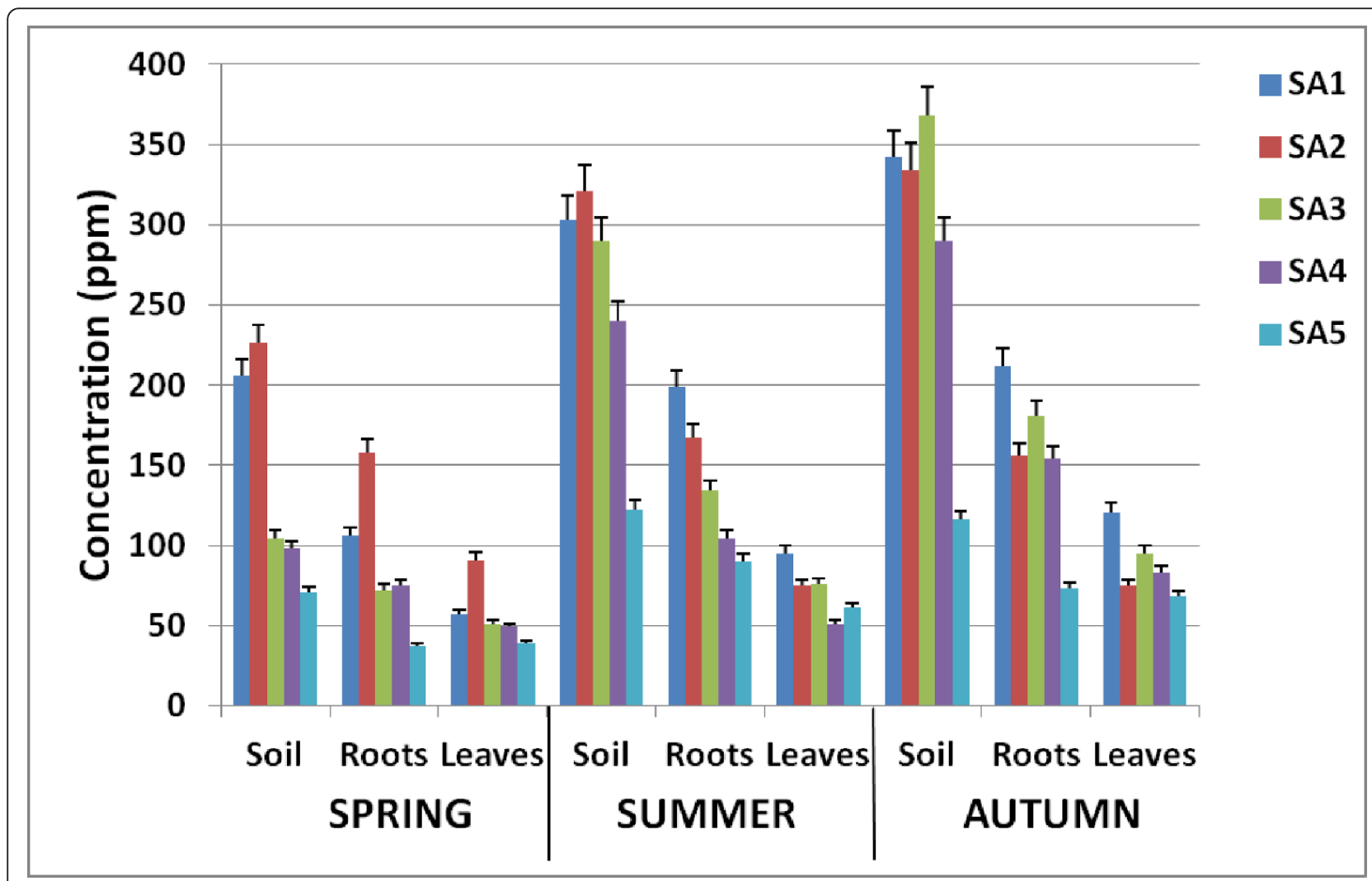

Figure 2 Heavy metals concentration in soil, roots and leaves as a function of sampling sites and seasons. Heavy metals concentrations in different seasons and in different sampling areas. Concentration in soil, root and leaves are also reported. As indicative example, Zn concentration in Plantago major L. is reported. 
Table 5 Heavy metals mean concentration for selected herbaceous plants. Concentrations of heavy metals contained in selected common plants. Data have been reported together with correlation coefficients in Figure 3.

\begin{tabular}{|c|c|c|c|c|}
\hline \multirow{3}{*}{$\mathrm{Pb}$} & \multicolumn{4}{|c|}{ Urtica dioica L. } \\
\hline & \multicolumn{2}{|c|}{ Mean concentration (ppm) } & \multicolumn{2}{|c|}{ Standard Deviation } \\
\hline & Soil & Leaves & Soil & Leaves \\
\hline SA1 & 708.7 & 24.7 & 180 & 4.7 \\
\hline SA2 & 759 & 21 & 272.2 & 2 \\
\hline SA3 & 399 & 14.3 & 169.2 & 1.5 \\
\hline SA4 & 225.3 & 12.7 & 71.1 & 1.5 \\
\hline \multirow[t]{2}{*}{ SA5 } & 178.7 & 10.3 & 37 & 1.5 \\
\hline & \multicolumn{4}{|c|}{ Taraxacum officinale L. } \\
\hline \multirow[t]{2}{*}{$\mathrm{Cu}$} & \multicolumn{2}{|c|}{ Mean concentration (ppm) } & \multicolumn{2}{|c|}{ Standard Deviation } \\
\hline & Soil & Leaves & Soil & Leaves \\
\hline SA1 & 131 & 46 & 5 & 9.5 \\
\hline SA2 & 134 & 42 & 15.6 & 3 \\
\hline SA3 & 121.3 & 36.7 & 29.7 & 2.5 \\
\hline SA4 & 85 & 24.3 & 37.7 & 1.5 \\
\hline SA5 & 63.7 & 16.3 & 36.8 & 7.1 \\
\hline
\end{tabular}

\begin{tabular}{ccccc}
\hline Mn & \multicolumn{2}{c}{ Mean concentration $(p p m)$} & \multicolumn{2}{c}{ Standard Deviation } \\
\hline & Soil & Leaves & Soil & Leaves \\
\hline SA1 & 656.7 & 55.3 & 104.7 & 26.8 \\
SA2 & 591 & 28 & 121 & 6.6 \\
SA3 & 647.3 & 48.3 & 118.4 & 21.8 \\
SA4 & 660 & 66.3 & 140 & 22.4 \\
SA5 & 582 & 30.3 & 150.3 & 6.4 \\
\hline
\end{tabular}

\begin{tabular}{ccccc}
\hline \multicolumn{5}{c}{ Trifolium pratense L. } \\
\hline Cu & Mean concentration $(p p m)$ & \multicolumn{2}{c}{ Standard Deviation } \\
\hline & Soil & Leaves & Soil & Leaves \\
\hline SA1 & 126.6 & 43 & 33.6 & 34 \\
SA2 & 102.4 & 37.7 & 33.6 & 19 \\
SA3 & 67.7 & 16.7 & 13.7 & 6.6 \\
SA4 & 72.3 & 23.1 & 29.3 & 5.9 \\
SA5 & 42.3 & 16.4 & 6.6 & 4.6 \\
\hline
\end{tabular}

Trifolium pratense L.

\begin{tabular}{ccccc}
\hline $\mathbf{P b}$ & \multicolumn{2}{c}{ Mean concentration $(p p m)$} & \multicolumn{2}{c}{ Standard Deviation } \\
\hline & Soil & Leaves & Soil & Leaves \\
\hline SA1 & 970.7 & 11 & 242.7 & 4.6 \\
SA2 & 990 & 10.3 & 330.3 & 4.7 \\
SA3 & 418.3 & 8 & 159.1 & 3.6 \\
SA4 & 204.7 & 4 & 127.8 & 0.1 \\
SA5 & 105.3 & 4 & 23.4 & 2.8 \\
\hline
\end{tabular}

some other sources, most likely of natural origin. In fact it has been reported that $\mathrm{Mn}$ present in soil comes for $89 \%$ from natural sources and only for the $11 \%$ from human activities [22]. Moreover, Mn gives rise to quite complex acid-base and redox equilibrium reactions in soil, depending on conditions (temperature, soil $\mathrm{pH}$ and structure, humidity, etc.) leading to a bio-distribution and bio-availability difficult to analyze in details without a widespread investigation that is beyond the scope of this work.

Taking into account the seasonal distribution of heavy metals in soil and the rainfall in Rome (Fig.1) we can hypothesize that higher temperatures and reduced rainfalls may determine a higher water evaporation leading to a higher accumulation (as dry weight) of metals with respect to spring or autumn. $\mathrm{Cu}$, and $\mathrm{Pb}$ seem to follow such behaviour, with a maximum concentration during summer (214 ppm and $1266 \mathrm{ppm}$, respectively). Zn reaches a maximum during autumn $(742 \mathrm{ppm})$ and $\mathrm{Mn}$ follows an opposite trend showing the lowest value during summer (449 ppm). Cr and Pd do not seem to be influenced by atmospheric conditions and their concentration remain relatively low and constant all over the year (between 15 and $45 \mathrm{ppm}$ for $\mathrm{Cr}$ and between 37 and $77 \mathrm{ppm}$ for Pd). Owing to the low Pd concentration and the almost equal distribution in all the sampling areas considered, we may conclude that the eventual release of this metal from catalytic converters is therefore negligible, at least in our study. Interestingly, We also noticed the same correlation between heavy metals accumulation in soil and the concentration of some selected metals found by Cardarelli et al. in lichens collected in Rome in the same periods [23]. The same increasing trend from spring to summer may be found for $\mathrm{Cu}, \mathrm{Zn}$ and $\mathrm{Pb}$, with maximum concentrations during summer (47 ppm for $\mathrm{Cu}, 260 \mathrm{ppm}$ for $\mathrm{Zn}$ and 180 for $\mathrm{Pb}$ ); on the contrary, $\mathrm{Mn}$ concentration decreases showing a minimum value $(32 \mathrm{ppm})$ in summer. The decrease of $\mathrm{Mn}$ concentration in lichens was attributed to a loose in vitality of these species, owing to the mediator effect of this metal in photosynthetic processes. Lichens are currently used as reliable bio-accumulators and bio-monitoring species to evaluate urban pollution (i.e., air quality). Since a similar behaviour was observed between air and soil pollutants, we can hypothesize the presence of a mechanism of transport from air to soil (most likely due to precipitations). However, our study suggests the presence of other mechanisms or events that should contribute to explain the reduced Mn content during summer. These events are not easily inferable and the collection of other data are needed to explain this behaviour.

\section{Heavy metals accumulation in plants}

In our study we have considered four different vegetal species (three herbaceous and one leguminous plants) in order to investigate the feasibility of employing them as useful and simple tools to monitor environmental pollution, and in particular soil pollution by heavy metals. We therefore investigated if these plants can be selective toward specific heavy metal and in order to minimize 


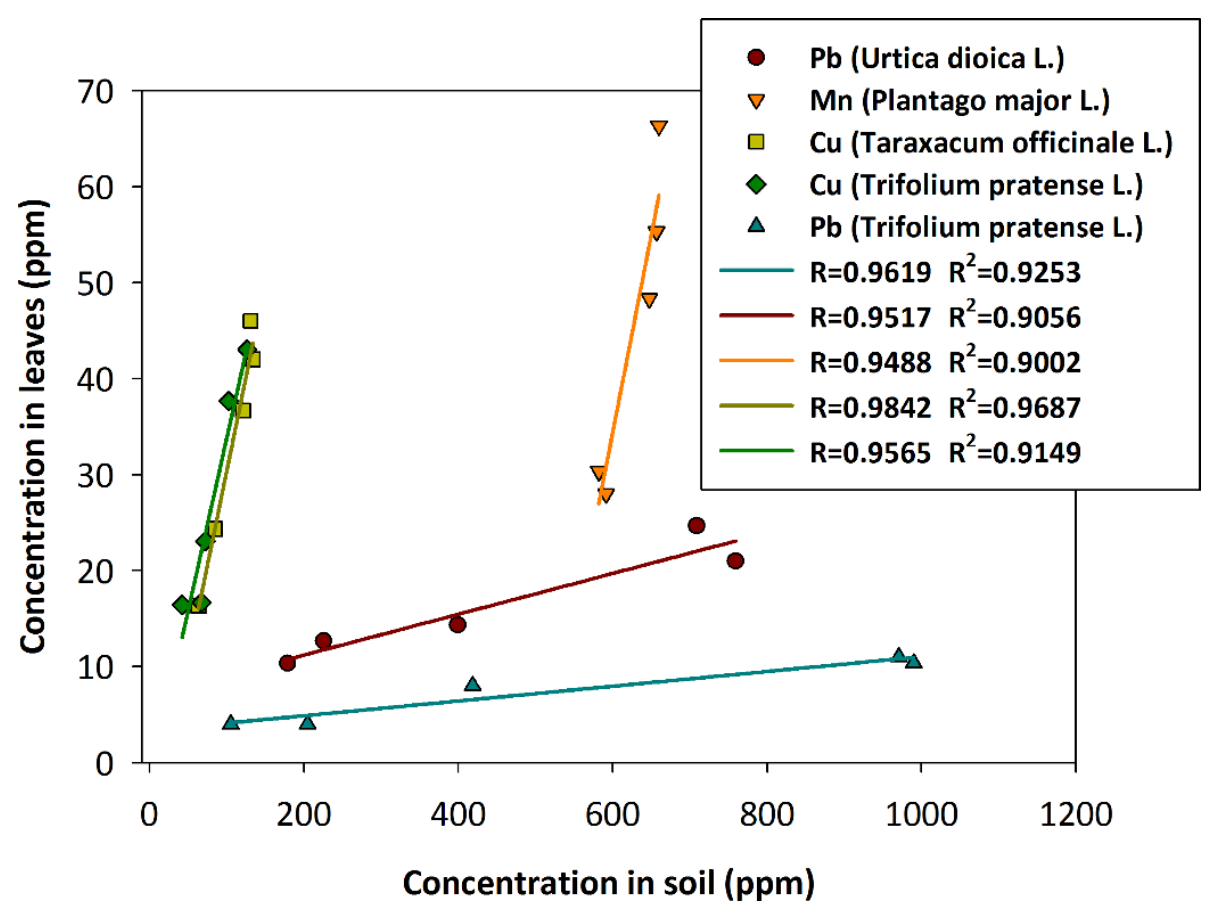

Figure 3 Correlation of heavy metal concentrations in soil and leaves. The correlation coefficient (expressed as $R$ and $R^{2}$ ) of the linear curve obtained after fitting the heavy metals concentrations in leaves against that of soil, in herbaceous and leguminous species.

variability in the analytical determination, we assessed the heavy metals concentration in three different seasons over the course of one solar year. From our extensive study, we found some direct correlations between the amount of heavy metals in soil and in the leaves of the selected plants (Fig. 3). Only $\mathrm{Cu}, \mathrm{Mn}$ and $\mathrm{Pb}$ display a good linear dependence on metal concentration in soil. In particular, both Taraxacum officinale L. and Trifolium pratense $L$. can accumulate $\mathrm{Cu}$ in their leaves in a linearly dependent manner respect to soil content. Additionally, the fraction of $\mathrm{Cu}$ accumulated by these two species is quite high (25-40\%) if compared to the amount present in soil. On the other hand, Plantago major L. can accumulate only small fractions of $\mathrm{Mn}(5-10 \%)$ in their leaves. Urtica dioica $L$. and Trifolium pratense L. are both able to accumulate $\mathrm{Pb}$ in their leaves even if at different percentages (10-20\% for Trifolium pratense $L$. and 30-60\% for Urtica dioica L.). For the latter two species, Urtica dioica $L$. represents the vegetal species that can accumulate the highest fraction of a dangerous heavy metal such as $\mathrm{Pb}$. The higher amount of $\mathrm{Pb}$ in the most polluted sampling areas (near trafficked roads) is a direct consequence of anthropogenic contribution, since in $1999 \mathrm{~Pb}$ was still added into fuels as an additive agent.

\section{Conclusions}

Our results demonstrate that common herbaceous and leguminous plants can be used as alternative and simple analytical tools that can be employed to monitor environmental pollution and in particular soil pollution by heavy metals. Other physicochemical parameters such as soil $\mathrm{pH}$, temperature, humidity, soil texture analysis, microbiological composition and soil redox potential, to cite only a few, have to be considered in order to deeply study the metal accumulation mechanisms by plants and employ them as efficient indicators of environmental pollution. Moreover, increasing the number of vegetal species it will be possible to find better indicators for different heavy metals, and suggest a panel of common plants to employ routinely in analytical determinations for environmental pollution monitoring.

\section{Acknowledgments}

Authors thank the Meteorological Centre of Rome, the Meteorological Centre of Milan (Centro Meteorologico Lombardo) and the Italian

Meteorological Society Onlus of Turin for having provided atmospheric data, Dr. Lorenzo Ciccarese for helpful suggestions and discussions and revision of the first manuscript, Dr. Fabiana Console for collection and revision of bibliography, and the Italian Ministry of University and Research (MIUR) for financial support.

This article has been published as part of Chemistry Central Journal Volume 6 Supplement 2, 2012: Proceedings of CMA4CH 2010: Application of

Multivariate Analysis and Chemometry to Cultural Heritage and Environment. The full contents of the supplement are available online at http://journal. chemistrycentral.com/supplements/6/S2.

\section{Authors' contributions}

DM collected soil and plants samples for ICP-MS analysis, prepared them and helped AG to perform the analytical determinations, AG acquired and analyzed data, GO contributed to the writing of the manuscript and revision, 
AM organized the experimental setting, supervised the work and wrote the manuscript.

\section{Competing interests}

The corresponding author confirms that any or all personal, employment or commercial affiliations, stock or equity interests or patent-licensing arrangements that could be considered to pose a financial conflict of interest regarding the submitted manuscript have been disclosed to the editor or in the manuscript.

Published: 2 May 2012

\section{References}

1. Kluge B, Wessolek G: Heavy metal pattern and solute concentration in soils along the oldest highway of the world - the AVUS Autobahn. Environ Monit Assess 2011, [Epub ahead of print].

2. Khan MN, Wasim AA, Sarwar A, Rasheed MF: Assessment of heavy metal toxicants in the roadside soil along the $\mathrm{N}-5$, National Highway, Pakistan. Environ Monit Assess 2011, 182(1-4):587-95.

3. Xia $X$, Chen $X$, Liu R, Liu H: Heavy metals in urban soils with various types of land use in Beijing, China. J Hazard Mater 2011, 186(2-3):2043-50.

4. Chen $X, X i a X$, Zhao $Y$, Zhang P: Heavy metal concentrations in roadside soils and correlation with urban traffic in Beijing, China. $J$ Hazard Mater 2010, 181(1-3):640-6.

5. Caggiano R, D'Emilio M, Macchiato M, Ragosta M: Ryegrass species as biomonitors of atmospheric heavy metals emissions. Fresenius Environ Bull 2001, 10(1):31-36.

6. D'Souza RJ, Varun M, Masih J, Paul MS: Identification of Calotropis procera L. as a potential phytoaccumulator of heavy metals from contaminated soils in Urban North Central India. J Hazard Mater 2010, 184(1-3):457-64.

7. Huang H, Gupta DK, Tian S, Yang XE, Li T: Lead tolerance and physiological adaptation mechanism in roots of accumulating and nonaccumulating ecotypes of Sedum alfredii. Environ Sci Pollut Res Int 2011, [Epub ahead of print].

8. Elekes CC, Dumitriu I, Busuioc G, Iliescu NS: The appreciation of mineral element accumulation level in some herbaceous plants species by ICPAES method. Environ Sci Pollut Res Int 2010, 17(6):1230-6.

9. Yanqun Z, Yuan L, Jianjun C, Haiyan C, Li Q, Schvartz C: Hyperaccumulation of $\mathrm{Pb}, \mathrm{Zn}$ and $\mathrm{Cd}$ in herbaceous grown on lead-zinc mining area in Yunnan, China. Environ Int 2005, 31(5):755-62.

10. Yanqun Z, Yuan L, Schvartz C, Langlade L, Fan L: Accumulation of Pb, Cd, $\mathrm{Cu}$ and $\mathrm{Zn}$ in plants and hyperaccumulator choice in Lanping lead-zinc mine area, China. Environ Int 2004, 30(4):567-76.

11. Vandenabeele WJ, Wood OL: The distribution of lead along a line source (highway). Chemosphere 1972, 1(5):221-226.

12. Nakayama SM, Ikenaka $Y$, Hamada $\mathrm{K}$, Muzandu $\mathrm{K}$, Choongo $\mathrm{K}$, Teraoka $\mathrm{H}$, Mizuno N, Ishizuka M: Metal and metalloid contamination in roadside soil and wild rats around a $\mathrm{Pb}-\mathrm{Zn}$ mine in Kabwe, Zambia. Environ Pollut 2011, 159(1):175-81.

13. Nam DH, Lee DP: Possible routes for lead accumulation in feral pigeons (Columba livia). Environ Monit Assess 2006, 121(1-3):355-61.

14. Harmanescu M, Alda LM, Bordean DM, Gogoasa I, Gergen I: Heavy metals health risk assessment for population via consumption of vegetables grown in old mining area; a case study: Banat County, Romania. Chem Cent J 2011, 5:64.

15. Williamson P, Evans PR: Lead: levels in roadside invertebrates and small mammals. Bull Environ Contam Toxicol 1972, 8(5):280-288.

16. Quarles HD III, Hanawalt RB, Odum WE: Lead in small mammals, plants and soil at varying distance from a highway. J Appl Ecol 1977, 11(3):937-949.

17. Sawidis T, Breuste J, Mitrovic M, Pavlovic P, Tsigaridas K: Trees as bioindicator of heavy metal pollution in three European cities. Environ Pollut 2011, 159(12):3560-70.

18. Gallagher FJ, Pechmann I, Bogden JD, Grabosky J, Weis P: Soil metal concentrations and vegetative assemblage structure in an urban brownfield. Environ Pollut 2008, 153(2):351-61.

19. Staszewski T, Lukasik W, Kubiesa P: Contamination of Polish national parks with heavy metals. Environ Monit Assess 2011, [Epub ahead of print].

20. Ots K, Mandre M: Monitoring of heavy metals uptake and allocation in Pinus sylvestris organs in alkalised soil. Environ Monit Assess 2011, [Epub ahead of print].
21. Moreno E, Sagnotti L, Dinarès-Turell J, Winkler A, Cascella A: Biomonitoring of traffic air pollution in Rome using magnetic properties of tree leaves. Atmos Environ 2003, 31(21):2967-2977.

22. Nriagu JO: A global assessment of natural sources of atmospheric trace metal. Nature 1989, 338:47-49.

23. Cardarelli E, Achilli M, Campanella L, Bartoli A: Monitoraggio dell'inquinamento da metalli pesanti mediante l'uso di licheni nella città di Roma. Inquinamento 1993, 35(6):56-63.

doi:10.1186/1752-153X-6-S2-S6

Cite this article as: Malizia et al:: Common plants as alternative analytical tools to monitor heavy metals in soil. Chemistry Central Journal 2012 6(Suppl 2):S6.

\section{Publish with ChemistryCentral and every scientist can read your work free of charge \\ "Open access provides opportunities to our colleagues in other parts of the globe, by allowing anyone to view the content free of charge." W. Jeffery Hurst, The Hershey Company.}

- available free of charge to the entire scientific community

- peer reviewed and published immediately upon acceptance

- cited in PubMed and archived on PubMed Central

- yours - you keep the copyright

Submit your manuscript here:

http://www.chemistrycentral.com/manuscript/<smiles>c1ccccc1</smiles>

ChemistryCentral 